\section{Resultados de un programa multidisciplinario para el control del hábito tabáquico}

\author{
PEDRO PEÑA ${ }^{1}$, MÓNICA ZAGOLIN ${ }^{1}$, MÓNICA ACUÑA ${ }^{2, a}$, \\ SANDRA NAVARRETE ${ }^{3, \mathrm{~b}}$, PILAR BUSTAMANTE ${ }^{3, \mathrm{~b}}$, CLAUDIO SUÁREZ ${ }^{4}$, \\ GABRIEL CAVADA ${ }^{5, \mathrm{c}}, \mathrm{M}$. PAZ BERTOGLIA ${ }^{6, \mathrm{~d}}$

\section{Results of a multidisciplinary program to quit smoking}

Background: Therapies to quit smoking are based on counseling, psychological therapy $(P T)$, nicotine replacement therapy, bupropion or varenicline. Aim: To report the results of a multidisciplinary program to quit smoking. Material and Methods: Patients aged 18 years or more, motivated to quit smoking were admitted in a program based in counseling and PT, with or without pharmacological therapy. They were assessed by telephone during one year of follow up. Patients with unstable psychiatric diseases were excluded. Results were considered as "successful" when patients maintained abstinence during the year of follow up. A logistic regression analysis was done to identify factors associated with treatment success. Results: Between 2005 and 2011, 198 patients aged $45 \pm 11$ years (56\% males), who smoked $31.5 \pm 20.6$ packages/year, were treated. Of these, 155 (78\%) were treated with varenicline, 26 (13\%) with bupropion and 17 (9\%) did not receive pharmacological therapy. One hundred sixty eight patients completed the year of follow up. In 82 (49\%), treatment was successful and was negatively associated with a history of depression (odds ratio $=4$ (95\% confidence intervals 1.23-38.33). The main side effects associated to varenicline and bupropion were nausea in 37 and 23\%, sleep disorders in 20 and 19\% and headache in 12 and 0\%, respectively. Conclusions: A multidisciplinary program to quit smoking achieved a 49\% of abstinence during a year of follow up.

(Rev Med Chile 2013; 141: 345-352).

Key words: Nicotine; Smoking; Tobacco; Tobacco use disorder.

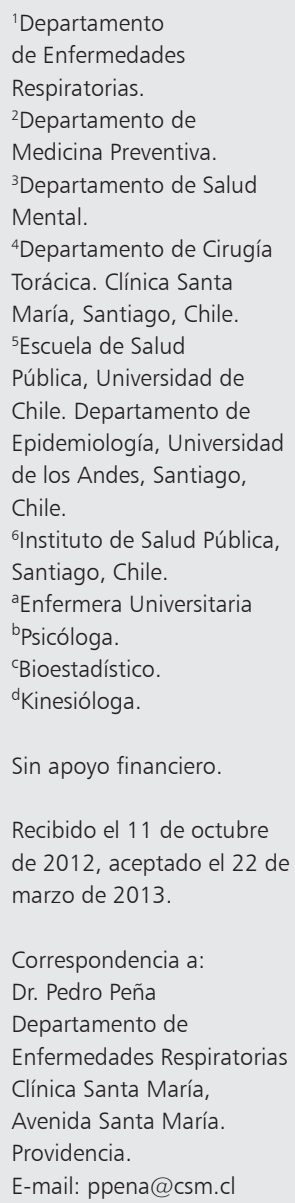

$\mathrm{E}$ 1 tabaquismo es un problema de primera prioridad sanitaria en Chile al igual que en el mundo. Constituye la principal causa de muerte evitable en los países desarrollados. El impacto de las estrategias impuestas a nivel mundial ha significado una disminución del hábito tabáquico (HT) en los países desarrollados pero un incremento en aquellos en desarrollo como es el caso de Chile cuya prevalencia reportada en los últimos años es de $41 \%^{1}$. El problema es extraordinariamente grave en el ambiente escolar, con cifras de hasta $40 \%$ en alumnos entre $8^{\circ}$ básico y $4^{\circ}$ medio $^{2}$, en mujeres embarazadas, en quienes se reportan cifras de tabaquismo de $28 \%$, que aumentan a $46,7 \%$ en adolescentes embarazadas ${ }^{3}$. También en el personal de salud se han reportado cifras alarmantes del orden de $40,7 \%{ }^{4}$. La prevalencia de tabaquismo en Chile es la más alta de Latinoamérica según el análisis efectuado por el grupo Platino ${ }^{5}$ y es una cifra preocupante si se considera que en el año 2004 se legisló al respecto, mediante la ley 20125 y se han generado esfuerzos relevantes tanto en atención primaria como terciaria para abordar el problema desde un punto de vista operativo y educativo. El impacto del tabaquismo en la salud a corto y largo plazo es superior al de cualquier 
otra condición y es por ello que la Organización Mundial de la Salud (OMS) lo ha denominado la epidemia de los tiempos modernos. El tabaco es factor causal de numerosas patologías dentro de las que destaca ocasionando $90 \%$ de las neoplasias de pulmón, $85-90 \%$ de la enfermedad pulmonar obstructiva crónica (EPOC) ${ }^{6,7}$ y $25 \%$ de la patología coronaria ${ }^{6}$. En Chile, el tabaco es factor causal de 15.000 muertes anuales que constituyen 17\% de todas las muertes ${ }^{8}$.

Las alternativas terapéuticas para abordar el HT se han ampliado desde 1980, en que sólo se disponía de sustitutos de nicotina (chicles, parches) hasta la era de 1990 en que se aprueba bupropión, la primera droga oral no nicotínica para el tratamiento de la adicción nicotínica (AN) y luego en el 2007 en que se aprueba vereniclina, un agonista parcial de los receptores nicotínicos. La efectividad de los sustitutos de nicotina, bupropión y vareniclina registrada luego de un año de seguimiento fluctúa dependiendo de la población estudiada y nivel de adicción entre $0-17 \%{ }^{9}, 15-30^{10}$ y $25-35 \%{ }^{11,12}$ respectivamente. Si bien los resultados mencionados podrían parecer insuficientes, es importante considerarlos en la perspectiva de una patología crónica, con potencial de recaída y de tan difícil control como cualquier otra condición crónica.

El tratamiento de la AN debe ser abordado en atención primaria por un equipo multidisciplinario, inserto en un programa de seguimiento y supervisión sistemática.

No existen reportes a nivel nacional del rendimiento a largo plazo de las diferentes terapias de la AN insertos en un programa multidisciplinario. A continuación, se presentan los resultados del programa instituido en Clínica Santa María (CSM).

\section{Material y Métodos}

Un estudio descriptivo de corte transversal fue iniciado en CSM en julio del año 2005 y se incluyeron todos los pacientes que ingresaron al Programa Antitabaco de la Clínica Santa María hasta agosto de 2011.

\section{Pacientes}

Todos los pacientes que consultan motivados por el abandono del hábito tabáquico son incorporados en el programa multidisciplinario de tratamiento y seguimiento de la AN y su información es registrada en una base de datos con el propósito de efectuar una evaluación epidemiológica y seguimiento sistemático durante un año. Son excluidos aquellos pacientes con enfermedad psiquiátrica inestable (depresión activa, síndrome bipolar, síndrome ansioso no tratado, situaciones de pánico, anorexia o bulimia, entre otras). Se excluyeron pacientes con insuficiencia renal en diálisis, con antecedente de epilepsia o traumatismo encéfalo craneano reciente y embarazadas, por las dificultades en la dosificación de vareniclina o bupropión y sus potenciales efectos tóxicos.

\section{Protocolo de trabajo}

El programa se compone de una evaluación médica inicial y controles posteriores en las semanas 4-8-24; una evaluación psicológica a cargo de psicólogas expertas en adicciones, que evalúan al paciente y lo incorporan en un plan de sesiones para terapia cognitivo-conductual en las semanas 2-4-6-8, de al menos 30 min de duración cada una. En concomitancia con estas sesiones, los pacientes son citados por la enfermera coordinadora del programa, quien efectúa una primera entrevista, registra datos epidemiológicos, hace entrega gratuita de un disco compacto de relajación y un manual instructivo de bolsillo, ambos de producción interna. En el manual se detallan aspectos relevantes del proceso de abandono y se especifica el tipo de dieta y actividad física que complementa la terapia específica de la AN.

En el momento del ingreso de cada paciente al programa se recolectaron las siguientes variables epidemiológicas: sexo, edad, estado civil, nivel educacional, edad de inicio del HT, número de cigarrillos consumidos al día, número de paquetes/ año (número de cigarrillos multiplicado por los años de consumo dividido por 20), comorbilidades, test de Fagerström para evaluar el nivel de adicción, los intentos previos de abandono y el peso. Todos los pacientes reciben consejería médica, terapia cognitivo conductual personalizada y según cada caso, terapia farmacológica, ya sea con sustitutos de nicotina, bupropión o vareniclina, en las dosis y metódica de inicio recomendadas por los fabricantes. Los pacientes son advertidos de los efectos adversos de cada alternativa y de los costos asociados que deben ser financiados por ellos mismos. De preferencia se recomendó vareniclina en primer lugar ya que es la droga con mejores resultados de efectividad y seguridad en los estudios randomizados comparativos y además 
es el inhibidor más específico de los receptores nicotínicos. En los pacientes que lo desearon o que su médico consideró apropiado, se optó por consejería exclusiva.

Los pacientes de común acuerdo con los profesionales, eligieron un día “ $D$ ” correspondiente al día de inicio de la abstinencia absoluta del hábito tabáquico. En los casos en que se prescribió terapia farmacológica con bupropion o vareniclina, el inicio de cualquiera de ellas fue 7 a 10 días antes del día “D” prefijado y la terapia se prolongó por 2-3 meses según cada caso.

\section{Seguimiento}

Luego del periodo inicial de terapia médica, de apoyo psicológico y de seguimiento médico estricto (primeros 2-3 meses) los pacientes fueron monitorizados por la enfermera coordinadora cada mes mediante llamadas telefónicas y visitas coordinadas hasta completar un año de seguimiento. Las llamadas y visitas con la enfermera no tienen costo adicional para el paciente y en ellas recibe asesoría y asistencia en las diversas fases del estudio y tratamiento, y ayuda logística para la obtención de consultas extra con médico o psicólogo ante cualquier potencial evento adverso o recaída. En cada entrevista telefónica se registró el nivel de consumo, molestias o eventos adversos y en la entrevista final del año, presencial, se registró el peso.

Para el análisis de efectividad sólo se utilizaron los datos de los pacientes que hubiesen completado un año de seguimiento y se utilizaron las siguientes definiciones operativas:

1. Éxito: se consideró en los casos en que la abstinencia fuera total (consumo de cigarrillos igual a cero) en el seguimiento de un año.

2. Fracaso: Se denominó a cualquiera de las siguientes situaciones:

2.1. Nunca abandono: pacientes que desde un inicio de terapia sea farmacológica o no farmacológica, persistieron fumando a un nivel igual o similar al previo.

2.2. Recaída: pacientes que lograron un éxito inicial de 2-3 meses, mientras se encontraban bajo terapia farmacológica y que luego de ello, en algún momento del seguimiento anual, reincidieron en el HT.

2.3. Disminución de dosis: pacientes que disminuyeron su consumo a menos de la mitad del consumo previo.

\section{Estadística}

Con el objetivo de conocer los factores que influyen en la cesación del hábito tabáquico se estudiaron diversas variables y sus relaciones con el éxito de la terapia, de forma univariada en primera instancia, y de forma multivariada luego, para evaluar su asociación conjunta. Se analizaron sólo los pacientes que habían completado un año de seguimiento. Se utilizaron promedios y medianas como medidas de tendencia central, porcentajes de frecuencias y percentiles como medidas de posición. Para probar la normalidad de los grupos de variables, se utilizó la prueba Shapiro-Wilk. Para comparar y realizar el análisis estadístico, se utilizó el $t$-test para variables numéricas continuas que siguen distribución normal. Como alternativa no paramétrica se utilizó el test de Wilcoxon RankSum. Para variables categóricas se realizó el Test de $\chi^{2}$ o el Test de Fisher, según corresponda. Se consideró estadísticamente significativo a un valor de $\mathrm{p}<0,05$. Para analizar la asociación conjunta entre cada una de las covariables y el resultado del programa (éxito o fracaso) se realizó un Modelo de Regresión Logística Multivariado, con los factores cuya significación fuera de al menos 0,25 (Criterio de Hosmer y Lemeshow) en el análisis univariado. Se analizaron los datos con el software estadístico STATA 11.

\section{Resultados}

Entre julio de 2005 y agosto de 2011, 198 pacientes completaron los criterios de inclusión para ser incorporados en el programa de tratamiento de la AN en CSM. La edad promedio fue de 45 \pm 11 años siendo 111 (56\%) hombres. El inicio del hábito se registra en promedio a los 16 años y $73,3 \%$ declara el antecedente de tabaquismo en los padres. La serie evaluada tiene un alto nivel de adicción nicotínica y más de la mitad de ellos tiene comorbilidades asociadas. Más de dos tercios de los pacientes ha intentado con algún método abandonar el hábito antes de consultar al programa y $36,7 \%$ declara el antecedente de haber tenido una depresión antes tratada. Las principales características epidemiológicas de los pacientes se ilustran en la Tabla 1.

Todos los pacientes recibieron consejería médica y terapia cognitivo conductual. Las terapias farmacológicas seleccionadas se distribuyeron en las siguientes frecuencias: 155 pacientes $(78,28 \%)$ 
Control del hábito tabáquico - P. Peña et al

\section{Tabla 1. Variables epidemiológicas de los pacientes que ingresaron al Programa de Control del Hábito Tabáquico}

\begin{tabular}{|c|c|c|}
\hline \multicolumn{3}{|c|}{ Características } \\
\hline \multicolumn{2}{|l|}{ Edad } & $45,13 \pm 11,23(18-73)$ \\
\hline \multirow[t]{2}{*}{ Sexo: } & Hombres & $111(56,06 \%)$ \\
\hline & Mujeres & $87(43,94)$ \\
\hline \multicolumn{2}{|c|}{ Edad de inicio del hábito } & $16,26 \pm 3,63(8-40)$ \\
\hline \multicolumn{2}{|c|}{$\mathrm{N}^{0}$ paquetes /año } & $31,48 \pm 20,61(3,73-110)$ \\
\hline \multicolumn{2}{|c|}{ Fagerström } & $5,41 \pm 2,07(0-10)$ \\
\hline \multicolumn{2}{|c|}{ Antecedente familiar } & $176,99(89,39 \%)$ \\
\hline \multicolumn{2}{|c|}{ Intentos previos } & $152(76,77 \%)$ \\
\hline \multicolumn{2}{|c|}{ Antecedente de depresión } & $73,24(36,99 \%)$ \\
\hline \multicolumn{2}{|c|}{ IMC basal* } & $25,18 \pm 4(16,5-35)$ \\
\hline \multicolumn{2}{|c|}{ Estado civil: casado/separado } & $119(60,1 \%) / 32(16,16 \%)$ \\
\hline \multicolumn{2}{|c|}{ Nivel educacional superior ${ }^{\ddagger}$} & $111(56,06 \%)$ \\
\hline \multicolumn{2}{|c|}{ Comorbilidad } & $105(53,03 \%)$ \\
\hline & Vascular $^{+}$ & $80(40,4 \%)$ \\
\hline & Bronquial $^{++}$ & $37(18,69 \%)$ \\
\hline Total & & $198(100 \%)$ \\
\hline
\end{tabular}

Los valores son expresados en Media y desviación estándar y entre paréntesis, los porcentajes o los límites mínimos y máximos según corresponda. ${ }^{*} \mathrm{IMC}=$ Índice de masa corporal ¥Nivel Educacional Superior: Más de 12 años de educación ${ }^{+}$Comorbilidad vascular: Cardíaca, cerebral o vascular periférica de extremidades. ${ }^{++}$Comorbilidad bronquial: Asma y enfermedad pulmonar obstructiva crónica (EPOC).

recibieron vareniclina, $26(13,13 \%)$ bupropion y $17(8,59 \%)$ optaron por recibir sólo consejería médica y psicológica.

En el momento de este reporte, 30 pacientes se encuentran aún en fases tempranas del seguimiento por lo que el análisis de efectividad se efectuó con los 168 que han sido seguidos por un año. De ellos, se logró abstinencia total (“éxito") en 82 pacientes $(48,81 \%)$, como se ilustra en la Figura 1.

El éxito de la terapia no dependió significativamente de la estrategia escogida, evidenciándose una frecuencia de éxito y no éxito de 46,9 y $53,1 \%$ para vareniclina, 46,2 y $53,8 \%$ para bupropion y 71,4 y $28,6 \%$ para consejería exclusiva respectivamente $(\mathrm{p}=0,658)$, aunque cabe destacar que el número de individuos que optó por terapias diferentes a vareniclina es muy pequeño para validar este tipo de comparaciones.
Tabla 2. Análisis Multivariado para detección de factores asociados con el éxito de la terapia

\begin{tabular}{|c|c|c|c|}
\hline Variable & OR & $95 \%$ IC & Valor-p \\
\hline Edad & 1 & $0,92-1,09$ & 0,839 \\
\hline Sexo & 0,35 & $0,06-2,0$ & 0,243 \\
\hline Estado Civil & 1,16 & $0,3-4,43$ & 0,826 \\
\hline Nivel Educacional & 3,31 & $0,83-13,12$ & 0,087 \\
\hline Antecedente Padre & 1,3 & $0,36-4,61$ & 0,679 \\
\hline Antecedente Madre & 0,86 & $0,22-3,34$ & 0,828 \\
\hline Fagerström & 2 & $0,93-4,29$ & 0,072 \\
\hline Intentos Previos & 0,76 & $0,38-1,54$ & 0,448 \\
\hline Antecedente depresión & 4 & $1,23-38,33$ & $0,049 *$ \\
\hline Comorbilidad & 1,14 & $0,31-4,14$ & 0,834 \\
\hline Fármaco & 1,30 & $0,4-4,18$ & 0,658 \\
\hline Paquetes/año & 1 & $0,96-1,04$ & 0,798 \\
\hline Edad de inicio & 0,92 & $0,8-1,02$ & 0,14 \\
\hline Intentos previos & 0,75 & $0,34-1,64$ & 0,47 \\
\hline IMC inicial & 1,02 & $0,97-1,08$ & 0,298 \\
\hline Trastorno sueño & 1,16 & $0,22-5,96$ & 0,857 \\
\hline Náuseas & 0,96 & $0,27-3,35$ & 0,951 \\
\hline Cefalea & 0,29 & $0,04-1,94$ & 0,206 \\
\hline Malestar Digestivo & 1,33 & $0,12-14,02$ & 0,809 \\
\hline
\end{tabular}

*Sólo el antecedente Depresión se asoció significativamente con el éxito de la terapia.

En el análisis univariado, presentan una asociación significativa con el éxito la cantidad de meses con medicamento $(\mathrm{p}=0,0171)$ y el antecedente de depresión $(\mathrm{p}=0,006)$ con un OR de prevalencia de 2,75 (IC 95\% 1,29-5,88). En el análisis multivariado de todos los factores epidemiológicos registrados, sólo el antecedente de depresión se asoció significativamente con el "No éxito" de la terapia $(\mathrm{p}=0,049)$ con un OR de prevalencia de 4(IC 95\% 1,23-38,33 (Tabla 2).

En relación al incremento de peso, los pacientes en la evaluación inicial presentaron un Índice de Masa Corporal (IMC) normal de 25,18 y luego de un año experimentaron un aumento neto de peso de 3,74 kg (IC 95\%: 2.41-5,07). El incremento de peso según terapia fue de $3.4 \mathrm{~kg}$ para vareniclina, 4 $\mathrm{kg}$ para bupropion y 5,27 kg para los que optaron por consejería exclusiva $(\mathrm{p}=0,527)$. 


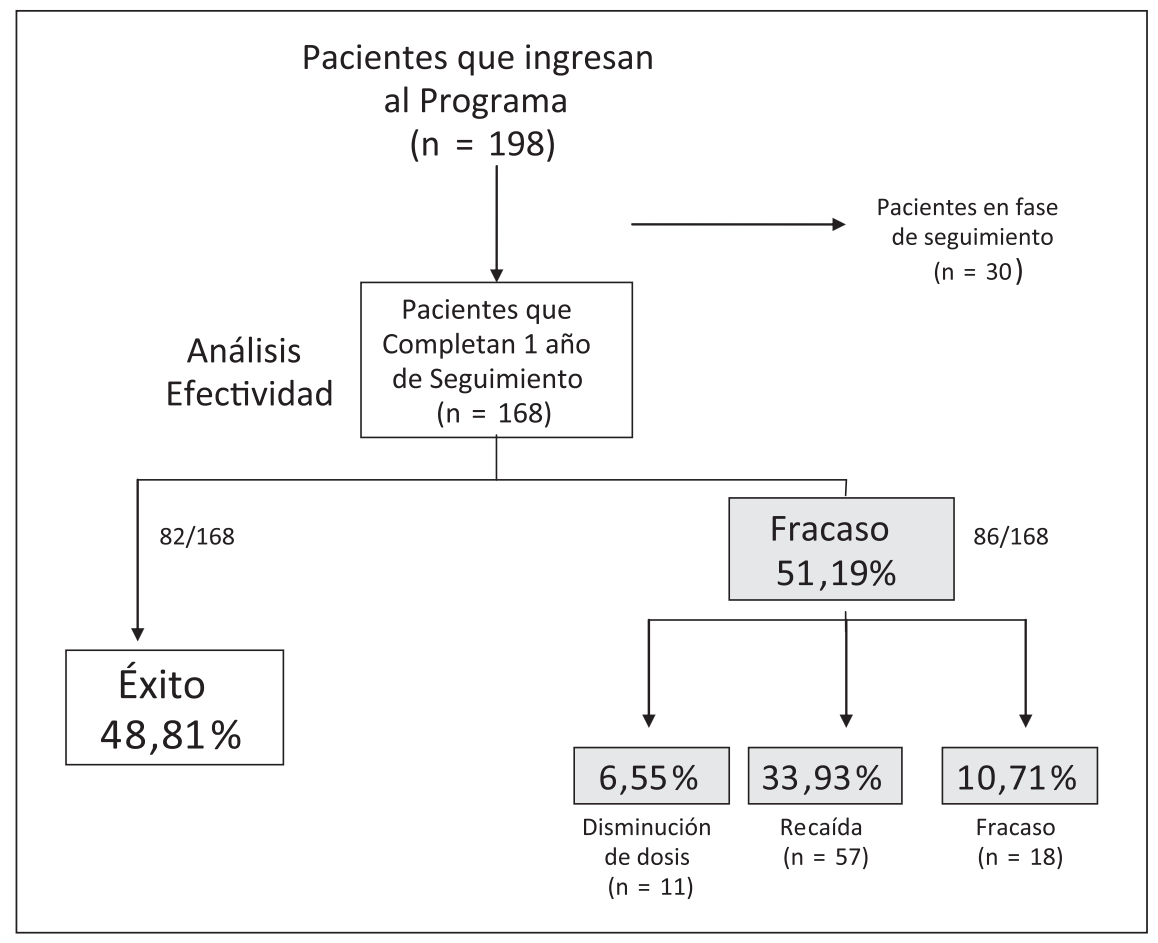

Figura 1. Gráfica de los Pacientes según resultado del Programa de Control del Tabaquismo.

Tabla 3. Principales efectos adversos según terapia farmacológica en los pacientes tratados

\begin{tabular}{|ccrcc|}
\hline Tratamiento & Náuseas & Alt.Sueño* & Cefalea & Total \\
\hline Vareniclina & $47(36,7 \%)$ & $26(20,3 \%)$ & $15(11,7 \%)$ & 128 \\
\hline Bupropión & $6(23 \%)$ & $5(19,2 \%)$ & 0 & 26 \\
\hline Total & $53(34,4 \%)$ & $31(20,1 \%)$ & $15(9,7 \%)$ & $154(100 \%)$ \\
\hline
\end{tabular}

*Alteraciones del sueño.

En 107 pacientes $(63,7 \%)$ se evidenció algún efecto adverso, sin embargo, sólo en dos casos $(1,2 \%)$ la terapia con vareniclina debió ser cambiada a bupropion en la primera semana debido a intolerancia digestiva. En el resto, las molestias fueron toleradas con medicación sintomática. Los principales efectos adversos reportados tanto para vareniclina, bupropion son: náuseas en 36,7 y $23 \%$; trastornos del sueño en 20,3 y 19,2\% y cefalea en 11,7 y $0 \%$ respectivamente (Tabla 3 ).

\section{Discusión}

Los resultados del programa antitabaco en CSM son satisfactorios en la perspectiva que se trata de una adicción crónica muy difícil de controlar y más aún, de revertir, sobre todo considerando el nivel de adicción de la población tratada. Un $48,81 \%$ de éxito al año es una cifra comparable a los resultados publicados en la literatura y satisfactoria para la población a la cual estamos enfrentados a atender. En una serie española de 1850 pacientes tratados y seguidos con una metódica similar, aunque con terapia de distribución gratuita, la frecuencia de abstinencia continua al año de seguimiento fue de $55 \%{ }^{13}$.

La población incorporada al programa destaca por constituir una población no sólo de alto nivel de AN sino con elevada prevalencia de comorbilidad asociada, principalmente vascular o respiratoria y con historia de depresión en 37\% de los casos. Este último factor se asoció signifi- 
cativamente con el fracaso del programa tanto en el análisis univariado como en el multivariado, con un OR de 4 en relación a la chance de fracasar. En el programa se excluyeron los pacientes con depresión activa, que puede ser del orden de 20\% según una serie de Clínica Mayo, ya que se sabe es un condicionante de fracaso ${ }^{14}$.

Respecto a la terapia utilizada en esta serie, todos los pacientes recibieron consejería médica, intervención psicológica basada en terapia cognitiva conductual y la mayor parte de ellos, terapia farmacológica. En la actualidad existe consenso que cualquier programa enfocado en el cese del tabaquismo debe ofrecer apoyo psicológico dirigido a tratar la dependencia psíquica y apoyo farmacológico para aliviar la dependencia física ${ }^{15-17}$.

La terapia más utilizada fue vareniclina, debido a que con ella se han reportado las mejores cifras de éxito en los ensayos randomizados ${ }^{11,12}$ y en el último meta-análisis de la Cochrane Data Base realizado sobre más de 10.300 pacientes, se encontró un OR de 2.31 a favor del fármaco frente a placebo ${ }^{18}$. La vereniclina actuaría por un doble mecanismo, ya sea estimulando los receptores $\alpha 4 \beta 2$ como agonista parcial, lo que eleva los niveles de dopamina y alivia los síntomas de privación y a través de la unión competitiva con los receptores, bloquea al menos parcialmente la activación dopaminérgica provocada por la nicotina en caso de una recaída ${ }^{19}$.

Los efectos adversos registrados son similares a los reportados en la literatura y entre ellos cabe destacar la presencia de náuseas, cefalea y trastornos del sueño $0^{10-12,17}$, ninguno de ellos de carácter serio. Las náuseas ocasionadas por vareniclina se pueden presentar en el inicio de la terapia y se atenúan en las siguientes semanas para luego desaparecer ${ }^{12}$.

Uno de los factores más difíciles de controlar y motivo de resistencia al inicio del proceso de abandono es el incremento de peso que ha sido involucrado en más de $40 \%$ de las recaídas ${ }^{20}$. Es sabido que un tercio del aumento de peso se debe a la disminución de la tasa metabólica basal y dos tercios al incremento del consumo calórico ${ }^{21}$. En nuestra serie se registró una ganancia de peso discreta de $3,71 \mathrm{~kg}$ que posiblemente sea factible de controlar con restricciones dietéticas e incremento de la actividad física.

Las principales fortalezas de este estudio son el seguimiento prolongado, el tratamiento bajo una metódica multidisciplinaria y la exclusión de pacientes con enfermedad psiquiátrica inestable como, por ejemplo, la depresión que se sabe es responsable de cerca de $25 \%$ de las recaídas ${ }^{22}$.

Las principales debilidades de este estudio apuntan hacia la falta de randomización y grupo control pero el diseño de este estudio pretende mostrar los resultados del funcionamiento de un programa en la práctica real de una serie en seguimiento. Otra debilidad que pudiera ser aludida es la ausencia de un método objetivo de validación de la abstinencia, ya sea con medición de metabolitos de la nicotina o la medición de monóxido de carbono espirado (CO) mediante cooxímetro. En relación a esto, existen trabajos que demuestran una alta correlación entre la respuesta verbal del paciente y la medición del CO espirado, confirmando la fiabilidad de los datos expresados por los pacientes que, en definitiva, no mienten cuando adhieren a los controles ${ }^{13,23}$. En un estudio español de 904 pacientes, no se observaron diferencias significativas entre la respuesta del paciente y la medición de $\mathrm{CO}^{24}$. En atención a los hallazgos obtenidos al comparar las respuestas de los pacientes y el nivel de CO espirado mediante el cooxímetro, se sugiere que el uso de este instrumento es prescindible pero constituye una estrategia motivacional importante que podría incidir en la adherencia al programa ${ }^{25}$. Por otro lado, dicha medición no está exenta de falsos negativos, ya que tiene una precisión variable ante un consumo previo a las $6 \mathrm{~h}$ de la medición y también es afectado por niveles ambientales de $\mathrm{CO}^{24}$. En nuestro programa, el contacto telefónico efectuado siempre por la misma persona que genera un lazo de comunicación asertiva desde el primer momento, constituye la base de la credibilidad de la información reportada por el paciente. Esto pudiera motivar a otros centros a reproducir metódicas y resultados similares ya que la implementación del programa no es compleja. Por otro lado, se ha demostrado que el apoyo por vía telefónica seriado a lo largo de la fase de abandono incrementa la eficacia de las otras estrategias implementadas ${ }^{26}$.

Es importante destacar que de todas formas existe más de $50 \%$ de los pacientes que fracasa, cuyo perfil no ha sido posible de identificar del todo aunque el hallazgo del antecedentes de depresión demostró estar significativamente vinculado a él, con una chance de fracasar de 4 veces con 
Control del hábito tabáquico - P. Peña et al

respecto al que no presenta dicho antecedente. Hallazgos similares se han comunicado en una serie uruguaya, en pacientes tratados con bupropión o terapias de reemplazo nicotínico ${ }^{27}$. Una terapia más prolongada en estos casos pudiera ser de mayor eficacia y respecto a esto ya existen comunicaciones que evidencian mejores resultados con la terapia prolongada de vareniclina $(24 \mathrm{sem})^{28}$.

\section{Conclusiones}

El programa tiene una efectividad global adecuada y sólo el antecedente de una depresión antes tratada incide negativamente en el éxito final. Consideramos que el enfrentamiento de la AN debe efectuarse en programas integrados y multidisciplinarios que permitan abordar al paciente desde una mirada global, incidiendo en la esfera psico-emocional, física, nutricional y funcional.

Esta comunicación quisiera contribuir como instrumento de motivación para los diferentes centros de atención primaria, para desarrollar estrategias multidisciplinarias en la atención del paciente con $\mathrm{AN} y$ a reproducir mejores resultados.

Agradecimientos: A todos los médicos de Clínica Santa María que nos han referido pacientes para terapia anti-tabaco.

\section{Referencias}

1. Consejo Nacional para el Control de Estupefacientes (CONACE). Ministerio del Interior: Gobierno de Chile. Octavo Estudio Nacional de Consumo de Drogas en Chile, 2008 en www.conace.cl

2. CONACE. Sexto Estudio Nacional de Drogas en Población Escolar de Chile, 2005, $8^{\circ}$ a $4^{\circ}$ medio. Informe final, Octubre de 2006.

3. Mallol J, Brandenburg D, Madrid R, Sempertegui F, Ramirez L, Jorquera D. Prevalencia de tabaquismo durante el embarazo en mujeres chilenas de bajo nivel socioeconómico. Rev Chil Enf Respir 2007; 23: 17-22.

4. Bello S, Soto M, Michalland S, Salinas J. Encuesta nacional de tabaquismo en funcionarios de salud. Rev Med Chile 2004; 132: 223-32.

5. Menezes AM, López MV, Hallal PC, Muiño A, PérezPadilla R, Jardin JR, et al. Prevalence of smoking and incidence of initiation in the Latin American adult population: the PLATINO study. BMC Public Health 2009; 9: 151.
6. US Department of Health and Human Services. The health consequences of smoking: a report of the Surgeon General, 2004. www.cdc.gov/tobacco/sgr/sgr_2004/ index.htm. Date last accessed: November 28, 2006. Date last updated: February 15, 2006.

7. Murray CJL, López AD. Alternative projections of mortality and disability by cause 1990-2020: Global Burden of Disease Study. Lancet 1997; 349: 1498-504.

8. Ministerio de Salud. Departamento de Estadística e información en Salud. 2006. http://deis.minsal.cl/deis/ tabaco/muertes.htm

9. Silagy C, Lancaster T, Stead L, Mant D, Fowler G. Nicotine replacement therapy for smoking cessation. Cochrane Database Syst Rev 2004; 3: CD000146.

10. Richmond R, Zwar N. Review of bupropion for smoking cessation. Drug and Alcohol Review 2003; 22: 201-20.

11. Gonzales D, Rennard SI, Nides M, Oncken C, Azoluay $\mathrm{S}$, Billing $\mathrm{CB}$, et al. Varenicline, an $\alpha 4 \beta 2$ nicotine acetylcholine receptor partial agonist vs sustained-release bupropion and placebo for smoking cessation. JAMA 2006; 296: 47-55.

12. Jorenby DE, Hays T, Rigotti NA, Azoulay S, Watsky EJ, Williams KE, et al. Efficacy of varenicline, an $\alpha 4 \beta 2$ nicotine acetylcholine receptor partial agonist, vs placebo or sustained-release bupropion for smoking cessation. JAMA 2006; 296 (1): 56-63.

13. Jiménez CA, Mayayo M, Cisero A, Amor N, Ruiz JJ, Fernández MC, et al. Resultados asistenciales de una unidad especializada en tabaquismo. Arch Bronconeumol 2009; 45 (11): 540-4.

14. Croghan I, Ebbert J, Hurt RD, Hays T, dale L, Warner N, et al. Gender differences among smokers receiving interventions for tobacco dependence in a medical setting. Add Behav 2009; 34: 61-7.

15. American Collage of Physicians. Smoking cessation. Ann Inter Med ITC 2007; 146: 2-16.

16. Aveyard P, West R. Managing smoking cessation. Br med Journal 2007; 335: 37-41.

17. Fiore MC, Jaen CR, Baker TR, Bailey WC, Benowitz N, Curry S, et al. Treating tobacco use and dependence. 2008 update. Clinical Practice Guideline. Rockville. US: Department of Health and Human Services .Public Health Service: 2008.

18. CH-Cahill K, Stead LF, Lancaster T. Nicotine receptor partial agonists for smoking cessation Cochrane Database Syst Rev 2011; 16: 2: CD006103.

19. Keating GM, Siddiqui MAA. Varenicline: a review of its use as and aid to smoking cessation therapy. CNS Drugs 2006; 20: 945-60.

20. Jiménez CA, Barrueco M, Solano S, Torrecilla M, Dominquez F, Díaz-Maroto JL, et al. Recomendaciones en 
el abordaje diagnóstico y terapéutico del tabaquismo. Documento de consenso. Arch Bronconeumol 2003; 39: 35-41.

21. Filozof C, Fernández Pinilla MC, Fernández-Cruz A. Smoking cessation and weight gain. Obes Rev 2004; 5: 95-103.

22. Breslau N, Johnson EO. Predicting smoking cessation and major depression in nicotine dependent smokers. Am J Pub Health 1999; 90: 1222-27.

23. Ockene J, Kristeller J, Goldberg R, Ockene I, Merrian P, Barret $\mathrm{S}$, et al. Somking cessation and severity of disease: the Coronary Artery Smoking Intervention Study. Health Psychol 1992; 11: 119-26.

24. Barrueco M, Jiménez Ruiz C, Palomo L, Torrecilla M, Romero P, Riesco JA. Veracidad de la respuesta de los fumadores sobre su abstinencia en las consultas de deshabituación tabáquica. Arch Bronconeumol 2005; 41 (3): $135-40$.

25. Cortí V, Moral M, López T, Boleda X, Gómez JC. Factores que incluyen en la cesación tabáquica: la cooximetría como instrumento de motivación. Prev Tab 2011; 13 (1): 14-9.

26. Stead LF, Lancaster T. Telephone counselling for smoking cessation. Cochrane library. N2. Oxford: Update software, 2001.

27. Llambí ML, Esteves E, Blanco ML, Barros M, Parodi C, Goja C. Factores predictores de éxito en el tratamiento del tabaquismo. Rev Med Urug 2008; 24: 83-93.

28. Jiménez Ruiz A, Cristobal M, López G, Cisero A, Mayayo M. Prev tab 2011; 13 (2): 65-9. 